\title{
IMPROPER MULTIPLE INTEGRALS
}

\author{
BY \\ R. G. D. RICHARDSON *
}

\section{INTRODUCTION.}

This paper is a continuation of a recent article by Professor Pierpont in this journal. $\dagger$ In that article attention is called to the fact that two methods for defining improper multiple integrals are available; $\ddagger$ one being that ordinarily followed; the other, that due to DE LA VALLEE-Poussin. In the present paper the theory will be developed from the latter point of view.

It is useful at the outset to introduce a notation that will obviate the difficulty that might arise from confusing the integrals defined by these different methods. Where any doubt is possible we shall denote $\|$ by $J$ the integral defined by the ordinary process, and by $\nabla$ that obtained by following the method of DE LA VALLEE-Poussin. It is evident at a glance that the integral $\nabla$ may, in all cases, be proved absolutely convergent. At the time of writing his article, Professor Prerpont had not been able to prove that his generalized integral J must necessarily be absolutely convergent, and hence a more general treatment seemed possible by following the older method of definition. We shall show in $\S 1$ that the integral $J$ is absolutely convergent. The discussion in the paper cited above may then be considerably simplified. After the definition in $\S 2$ of the improper multiple integral $\mathbf{\nabla}$, it is proved that the existence of either $\mathbf{J}$ or $\nabla$ is a necessary and sufficient condition for the existence of the other, and that both methods lead to the same result. In $\$ 3$ various tests for the convergence of singular integrals are outlined.

In this paper we shall regard the field of integration as limited. In a later paper various conditions for continuity, inversion of the order of integration, and reduction of multiple integrals to multiply iterated integrals will be discussed for both finite and infinite fields. In general it will be assumed that, in any partial aggregate in which the integrand is limited, the integral exists.

\footnotetext{
* Presented to the Society (New York), December 2A, 1905. Received for publication February 5, 1906. 174.

†Transactions of the American Mathematioal Society, vol. 7 (1906), pp. 153-

$\ddagger$ Loc. cit., p. 153, 81 .

II The letters $\mathrm{J}$ and $\mathbf{\nabla}$ are chosen to suggest respectively the names of the authora, JORDAN and VALLÉE-Poussin, who introdnced these methods for less geueral cases.
} 
In nearly all cases, however, the reasoning will hold for an upper or a lower integral. In any instance where the notation is not explained, the reader is referred to the paper cited above, or to a recent treatise* on the theory of functions by the same author.

\section{§1. Absolute convergence of the integrals defined by the first method.}

Before attacking the main problem we must prove some preliminary theorems. When the proof is simple, details are sometimes omitted.

Theorem 1. Let $f$ be a limited function of $m$ variables $x_{1}, x_{2}, \ldots, x_{m}$ defined over a limited aggregate $\mathfrak{A}$. Let $C$ be a constant. Then

$$
\overline{\int_{x}}(f+C)=\bar{\int}_{\mathfrak{x}} f+\int_{\mathfrak{x}} C .
$$

This follows at once from the relation $\max (f+C)=\max f+C$ in any cell $\delta_{\kappa}$ of the division $\Delta$.

Theorem 2 . Let $f \geqq 0$ be limited and defined over a limited aggregate $\mathfrak{A}$. Let $\mathfrak{B}$ and $\mathfrak{C}$ be partial aggregates of $\mathfrak{A}$, not necessarily unmixed, such that $\mathfrak{A}=\mathfrak{B}+\mathfrak{C}$. Then

$$
\overline{\int_{\mathfrak{x}}} f \leqq \overline{\int_{\mathfrak{B}}} f+\bar{\int}_{\mathfrak{E}} f .
$$

For, $\overline{\mathfrak{A}}_{\delta} \leqq \overline{\mathfrak{V}}_{\delta}+\overline{\mathfrak{E}}_{\delta}$. Hence if $M_{\kappa}$ is the maximum of $f$ in the cell $\delta_{\kappa}$,

$$
\sum_{k} M_{\kappa} \delta_{\kappa} \leqq \sum_{\sharp} M_{\kappa} \delta_{\kappa}+\sum_{\mathbb{E}} M_{\kappa} \delta_{\kappa} .
$$

Passing to the limit $\delta=0$, the theorem is proved.

Theorem 3. Let $\mathfrak{B}$ be any limited aggregate, and $\mathfrak{C}$ a partial aggregate of $\mathfrak{B}$. If any improper limiting points of $\mathfrak{E}$ lie in $\mathfrak{B}$, adjoin them to $\mathfrak{E}$ and call the resulting agqregate $C$. Let $\Delta_{1}, \Delta_{2}, \ldots$ be a series of superimposed divisions whose norms $\delta_{1}, \delta_{2}, \ldots$ converge to zero. Denote by $\mathfrak{B}_{\kappa}$ all points of $\mathfrak{B}$ lying in those cells of $\Delta_{k}$ which contain at least one point of $C$. Since $\mathfrak{B}_{\kappa}$ contains $C$ we may write $\mathfrak{B}_{\kappa}=C+B_{\kappa}$. Then

$1^{\circ}$. The aggregates $B_{1}, B_{2}, \ldots$ are such that $B_{n-1} \geqq B_{n}$.

$2^{\circ}$. The aggregates $B_{1}, B_{2}, \ldots$ have no point in common.

$3^{\circ}$. $\operatorname{Lim}_{n=\infty} \bar{B}_{n}=0$.

To prove $1^{\circ}$ note that, since the divisions are superimposed, each aggregate $\mathfrak{P}_{n-1}$ contains at least all of $\mathfrak{B}_{n}$. As $C$ does not change with the division, $B_{n-1} \geqq B_{n}$.

Suppose now $B_{1}, B_{2}, \ldots$ have a point $P$ in common; $P$ cannot be a limiting point of $C$, for $P$ lies in $B_{1}$ and hence in $\mathfrak{B}_{1}$ and in $\mathscr{P}$. But all limiting points

* Lectures on the Theory of Functions of Real Variables, vol. 1, Ginn \& Co. (1905). This will be referred to as Lectures. 
of $C$ lying in $\mathscr{B}$, lie in $C$ and cannot therefore be in any $B_{\kappa}$. Hence for $\rho$ sufficiently small, no poiut of $C$ and hence no point of $\mathbb{C}$ lies in $D_{\rho}(P)^{*}$, the domain of $P$ of norm $\rho$. Now if $\nu$ be taken sufficiently large, every point of that cell of the division $\Delta_{n}, n \geqq \nu$, containing the point $P$ must be entirely within $D_{\rho}(P)$. But this cell contains at least one point of $\mathfrak{C}$, which is a contradiction. The theoren $2^{\circ}$ then follows.

To prove $3^{\circ}$, we assume on the contrary, $\lim _{n=\infty} \bar{B}_{n}=\beta>0$. Then $\bar{B}_{1} \geqq \bar{B}_{2} \geqq \ldots \geqq \beta$; i. e., there are points in common to this series, no matter how great $n$ is taken. This contradicts $2^{\circ}$, and the theorem is proved.

THEOREM 4. Let $f$ be a limited function defined over a limited aggregate $\mathfrak{A}$. Let $\mathfrak{I}$ be a partial aggregate of $\mathfrak{A}$ such that $\overline{\mathfrak{B}}=\overline{\mathfrak{A}}$. Let $f$ be positive in $\mathfrak{B}$, and negative or zero elsewhere in $\mathfrak{A}$. Then

$$
\overline{\int_{x}} f=\int_{z}^{\bar{y}} f .
$$

Every cell containing a point of $\mathfrak{A}$ contains a point of $\mathfrak{Y}$, for otherwise $\overline{\mathfrak{Z}}<\overline{\mathfrak{A}}$. Hence

$$
\int_{\mathfrak{x}} f=\lim \sum_{*} M_{\kappa} \delta_{\kappa}=\lim \sum_{y} M_{\kappa} \delta_{\kappa}=\int_{y} f .
$$

Theorem 5. Let $f$ be limited and defined over a limited aggregate $\mathfrak{A}$. Let $\mathfrak{B}$ denote the points of $\mathfrak{A}$ where $f>0$. Let $g=f$ in $\mathfrak{B}$ and let $g=0$ at other points of $\mathfrak{A}$. Then

$$
\overline{\int_{x}} g=\int_{B} f .
$$

For let $\Delta$ be a division of space of narm $\delta$. Let $\delta$, denote cells containing points of $\mathfrak{B}$, while $\delta_{\kappa}$ denotes cells containing points of $\mathfrak{U}$ but none of $\mathfrak{Z}$. Then, since $g_{k}=0$,

$$
\sum_{\star} g \delta=\sum g_{\imath} \delta_{\imath}+\sum g_{k} \delta_{k}=\sum_{\nexists} f_{\imath} \delta_{\imath} .
$$

Passing to limit $\delta=0$, the theorem is proved.

It is now possible to prove the main theorem, which is a generalization of theorem 21. $\dagger$ There it is proved for relatively measurable fields; here all restrictions on the field are removed.

TheOREM 6. Let $f\left(x_{1}, x_{2}, \ldots, x_{m}\right)$ be defined over any limited aggregate A. If

$$
\overline{\int_{x}} f^{\prime}, \quad \int_{x} f
$$

are both convergent, they are absolutely convergent.

* For further definition of the symbol, see Lectures, p. 153.

† Loc. cit., p. 164. 
Let

and

$$
g=f \text { when } f>0, \quad g=0 \text { when } f \leqq 0
$$

$$
h=-f \text { when } f<0, \quad h=0 \text { when } f \geqq 0 \text {. }
$$

Then $|f|=g+h$. The upper integral of $|f|$ will be convergent if the upper integrals of $g$ and $h$ converge. We shall show first that for $\epsilon>0$ we can find $\delta>0$, such that, if $\mathfrak{B}$ is an aggregate exterior to the aggregate $I$ of singular points, and $\overline{\mathfrak{B}}<\delta$ then

$$
\left|\int_{y} g\right|<\epsilon .
$$

By theorem 5 * we can take $\delta$ such that, if $\mathfrak{B}_{\kappa}$ is a partial unmixed aggregate of $\mathfrak{B}$,

$$
\left|\bar{\int}_{x_{k}} f\right|<\frac{\epsilon}{3} \text {. }
$$

Denote by $\mathfrak{C}$ the points of $\mathfrak{B}$ where $f>0$. Adjoin to $\mathfrak{C}$ all its improper limiting points and call the resulting aggregate $C$. Then $\dagger \bar{C}=\overline{\mathbb{C}}$. Let $\Delta_{1}, \Delta_{2}, \ldots$ be a set of superimposed divisions whose norms converge to zero. Denote by $\mathfrak{B}_{k}$ all points of $\mathfrak{B}$ in the cells of $\Delta_{k}$ which contain at least one point of $C^{\gamma}$. Then $\mathfrak{B}_{\kappa}=C+B_{\kappa}$. Choose a constant $M$ such that $f+M>0$ for all points of $\mathfrak{B}$. Then by theorem 1

$$
\overline{\int_{*_{k}}}(f+M)=\int_{x_{k}}^{\bar{s}} f+\int_{\boldsymbol{x}_{k}} M .
$$

Also by theorem 2 and Lectures, $\S 729,6$,

or

$$
\overline{\int_{C}}(f+M) \leqq \overline{\int_{*_{k}}}(f+M) \leqq \overline{\int_{C}}(f+M)+\int_{B_{k}}^{-}(f+M),
$$

Similarly

$$
0 \leqq \overline{\int_{*_{k}}}(f+M)-\overline{\int_{c}}(f+M) \leqq \overline{\int_{B_{k}}}(f+M) \text {. }
$$

$$
0 \leqq \int_{\mathbb{R}_{k}}^{\bar{R}} M-\int_{C} M \leqq \int_{B_{\kappa}} M .
$$

From theorem 3, $\nu$ may be chosen so large that for $n \geqq \nu, \bar{B}_{n}$ is less than any assigned quantity. Hence

$$
\int_{B_{n}}^{\bar{M}} M<\frac{\epsilon}{\overline{3}}, \quad \int_{B_{n}}^{\bar{n}}(f+M)<\frac{\epsilon}{\overline{3}} .
$$

\footnotetext{
* Loc. cit., p. 159.

† Lectures, p. 522, § 715.
} 
Subtracting (4) and (5) and using (6) and (3), we have

and by use of (2),

$$
\left|\int_{n_{n}} f-\int_{C}^{-} f\right|<\frac{2 \epsilon}{3},
$$

$$
\int_{c} f<\epsilon .
$$

But by theorems 4 and 5 ,

Finally from (8) and (9),

$$
\overline{\int_{C}} f=\int_{\mathfrak{G}}^{\bar{c}} f=\int_{0}^{-} g .
$$

$$
\int_{8}^{\bar{g}} g<\epsilon .
$$

In an analogous manner it is shown that

Then

$$
\overline{\int_{0}} h<\epsilon .
$$

$$
\int_{\mathfrak{g}}|f| \leqq \int_{刃}|f|=\int_{\circledast}(g+h)<2 \epsilon .
$$

§2. A second method for defining improper multiple integrals.

Criteria for convergence.

The method followed in this new definition has as its foundation an outline given by DE LA VALLEE-Poussin.* Although the increase in the number of variables is an extension, the chief generalization is in regard to the field. While he expressly assumes that the contour in two dimensions is a curve $C$ which is cut but a finite number of times by a line parallel to one of the axes, we put no conditions whatever on the boundary, except to assunne that it is of content zero.

Until it has been proved that the integral defined by this method is identical with that discussed in $\S 1$, the symbols $J$ and $\nabla$ will be used for the integrals defined by the first and second methods; later, this auxiliary notation will be unnecessary.

If $f$ is both positive and negative in $\mathfrak{A}$, we define new functions as follows:

and

$$
\phi=0 \text { when } f \leqq 0, \quad \phi=f \text { when } f>0
$$

$$
\psi=0 \text { when } f \geqq 0, \quad \psi=|f| \text { when } f<0 .
$$

\footnotetext{
* Journal de Mathématiques, ser. 4, vol. 8 (1892), p. 421.
} 
Then $f=\phi-\psi$. Take $\lambda_{1}, \lambda_{2}$ arbitrarily large and define the auxiliary functions $\phi_{\lambda_{1}}, \psi_{\lambda_{2}}$.

and

$$
\phi_{\lambda_{1}}=\phi \text { for } \phi<\lambda_{1}, \quad \phi_{\lambda_{1}}=\lambda_{1} \text { for } \phi \geqq \lambda_{1}
$$

$$
\psi_{\lambda_{2}}=\psi \text { for } \psi<\lambda_{2}, \quad \psi_{\lambda_{2}}=\lambda_{2} \text { for } \psi \geqq \lambda_{2} .
$$

The corresponding function of $f$ will be denoted by the symbol $f_{\lambda_{1} \lambda_{2}}=\phi_{\lambda_{1}}-\psi_{\lambda_{2}}$. For any $\lambda_{1}$ and $\lambda_{2}$, the function $f_{\lambda_{1} \lambda_{2}}$ possesses an integral (definite, upper, or lower) and is a function of $x_{1}, \ldots, x_{m}$ and of the parameters $\lambda_{1}, \lambda_{2}$. If now

$$
\lim _{\lambda_{1}=\infty, \lambda_{2}=\infty} \nabla_{*} f_{\lambda_{1} \lambda_{2}}
$$

exists and is finite, we shall define our integral of $f$ as follows :

$$
\lim _{\lambda_{1}, \lambda_{2}=\infty} \nabla_{: f_{1}} f_{\lambda_{1}, \lambda_{2}}-\mathbf{\nabla}_{x} f \cdot *
$$

Theorem 7. Let $f \geqq 0$ be integrable in $\mathfrak{A} . \quad$ Let $\mathfrak{B}$ be a partial aggregate of $\mathfrak{A}$. Then the integral over $\mathfrak{B}$ exists and

$$
\mathbf{\nabla}_{\mathrm{x}} f \leqq \mathbf{\nabla}_{\mathrm{M}} f \text {. }
$$

For any $f_{\lambda}$ we know the integral over $\mathfrak{B}$ exists; and

$$
\nabla_{y,} f_{\lambda} \leqq \nabla_{*} f_{\lambda}<\nabla_{*} f .
$$

The expression on the left is a monotone increasing function of $\lambda$ and is limited by the expression on the right which is constant. Hence the integral over $\mathfrak{B}$ converges.

Three other theorems are stated below; the proof of each consists of a simple passage to the limit.

THEOREM 8. Let $\mathfrak{B}$ and $\mathfrak{C}$ be unmixed partial aggregates of $\mathfrak{A}, \mathfrak{B}+\mathfrak{C}=\mathfrak{A}$. If $f$ is integrable in $\mathfrak{B}$ and in $\mathbb{C}$, then $\mathbf{v}_{\mathfrak{x}} f=\mathbf{\nabla}_{\mathfrak{\Psi}} f+\mathbf{\nabla}_{\mathfrak{G}} f$.

TheоReм 9. Let $f$ and $g$ be integrable in $\mathfrak{A}$. Then $f+g$ is integrable in $\mathfrak{A}$ and $\nabla_{\mathfrak{x}}(f+g)=\mathbf{\nabla}_{\mathfrak{x}} f+\nabla_{\mathfrak{x}} g$.

ThEOREM 10 . Let $f \geqq 0, g \geqq 0$ be two functions integrable in $\mathfrak{A}$. Then $\mathbf{v}_{\mathrm{*}}(f+g) \geqq \mathbf{\nabla}_{\mathrm{M}} f \geqq \mathbf{\nabla}_{\mathrm{Y}} f_{\lambda}^{\prime}$.

* As an illustration of the metho ls used in this definition, we may calculate the volume of the solid generated by turning the Witch of Agnesi, $z^{2}=4 a^{2}(2 a-y) / y$, about its asymptote as axis. Here $\mathfrak{A}$ is a circle in the $x y$-plane having its center at the origin. The function which we integrate to find the volume has an infinite discontinuity at the origin. Pass planes parallel to the $x y$-plane at the distances $\lambda_{1}$ above and $\lambda_{2}$ below. The volume of this truncated solid may be computed by integrating $f_{\lambda_{1}} \lambda_{2}$,

$$
\nabla: x f_{\lambda_{1} \lambda_{2}}=\frac{8 a^{4} \pi \lambda_{1}}{4 a^{2}+\lambda_{1}^{2}}+4 a^{3} \pi \tan ^{-1} \frac{\lambda_{1}}{2 a}+\frac{8 a^{4} \pi \lambda_{2}}{4 a^{2}+\lambda_{2}^{2}}+4 a^{3} \pi \tan ^{-1} \frac{\lambda_{2}}{2 a} .
$$

Passing to the limit for $\lambda_{1}, \lambda_{2}=\infty$, we get $4 \pi^{2} a^{3}$ as the volume of the solid. 
ThenRem 11. In order that the integral of f exist, it is necessury and sufficient that the integrals of $\phi$ and $\psi$ exist.

With the notation $f_{\lambda_{\mathrm{c}} \lambda_{\kappa}}=\phi_{\lambda_{\mathrm{\iota}}}-\psi_{\lambda_{\kappa}}$,

$$
\left|\nabla f_{\lambda_{1} \lambda_{2}}-\nabla f_{\lambda_{3} \lambda_{4}}\right|<\frac{\epsilon}{2}, \quad\left|\nabla f_{\lambda_{1} \lambda_{2}}-\nabla f_{\lambda_{3} \lambda_{5}}\right|<\frac{\epsilon}{2}, \quad\left(\lambda_{1}, i_{2}, \lambda_{3}, i_{4}, i_{3}>R\right) .
$$

By subtraction,

$$
\left|\nabla f_{\lambda_{3} \lambda_{4}}-\nabla f_{\lambda_{3} \lambda_{6}}\right|=\left|\nabla \phi_{\lambda_{3}}-\nabla \psi_{\lambda_{4}}-\nabla \phi_{\lambda_{8}}+\nabla \psi_{\lambda_{5}}\right|=\left|\nabla \psi_{\lambda_{4}}-\nabla \psi_{\lambda_{6}}\right|<\epsilon .
$$

Hence $\nabla \psi$ exists. Similarly $\nabla \phi$ exists and the condition is necessary.

To prove the sufficiency of the condition we have, by theorems 9 and 10 , $\nabla(\phi+\psi)=\nabla \phi+\nabla \psi \geqq \nabla \phi_{\lambda}$. Now from this relation it is evident that $\mathbf{\nabla} \phi_{\lambda}$ is a limited monotone function. Then $\nabla \phi$ exists, and in a like manner it is shown that $\nabla \psi$ exists.

Theorem 12. In order that the integral $\nabla f$ exist, it is necessary and sufficient that $\boldsymbol{\nabla}|f|$ exist.

Since $f=\phi-\psi$ and $|f|=\phi+\psi$, this follows at once from the preceding theorem.

Theовем 13. In order that $\nabla_{q} f$ be convergent it is necessary and sufficient that, given $\epsilon>0$, we can find $\delta>0$, such that $\left|\nabla_{\sharp} f\right|<\epsilon$, for $\bar{\jmath}<\delta$, where $\mathfrak{B}$ is a partial aggregate of $\mathfrak{A}$.

It is necessary. For take $\lambda$ so large that $0<\nabla_{\mathrm{Y}}|f|-\nabla_{\mathrm{Y}}|f|_{\lambda}<\epsilon / 2$ and a fortiori

$$
0 \leqq \nabla_{\mathfrak{y}}|f|-\nabla_{\mathbb{y}}|f|_{\lambda}<\frac{\epsilon}{2}
$$

Since now $\lambda$ is fixed and $|f|_{\lambda}$ is a limited function, choose $\overline{\mathfrak{B}}$ so small that

Adding (1) and (2)

$$
0 \leqq \nabla_{\mathfrak{x}}|f|_{\lambda}<\frac{\epsilon}{2}
$$

$$
\left|\nabla_{x} f\right| \leqq \nabla_{*}|f|<\epsilon .
$$

The pronf that the condition is sufficient offers no special difficulty.

THEOREM 14. Let $\mathfrak{B}_{1}, \mathfrak{B}_{2}, \ldots$ be partial aggregates of $\mathfrak{A}$ such that. $\lim _{n=\infty} \overline{\mathfrak{P}}_{n}=\overline{\mathfrak{A}}$. If $\nabla_{\mathfrak{x}_{n}}|f|$ does not increase indefinitely when $n$ becomes infinite, the integral of $f$ over $\mathfrak{A}$ is absolutely convergent.

THEOREM 15. Let $\mathfrak{A}$ be a limited aggregate and $\mathfrak{B}$ a partial aggregate of $\mathfrak{A}$, mixed or unmixed. If the integral of $f$ exists in $\mathfrak{A}$ it exists in $\mathfrak{B}$.

Since $f$ is integrable in $\mathfrak{A}$ it is absolutely integrable. From theorem 7 it is evident that the integral of $|f|$ exists in $\mathfrak{B}$. Hence $f$ is integrable by theorem 12.*

* The various properties of integrals may be derived with great facility for the generalized integral by using this new definition. In many cases both definitions may be used in the same 
Having developed these criteria for the integrals $\nabla$, the relation to those * defined by Professor Pierpont may now be investigated.

THEOREM 16. If the improper multiple integral $\mathrm{J}$ exists, then $\nabla$ exists and $\mathbf{J}=\mathbf{\nabla}$.

Assume first that $f \geqq 0$. Then, by definition, there exists a division of norm $\delta$ such that

$$
0 \leqq \mathrm{~J}_{\mathrm{x}^{\prime} \delta} f=\mathrm{J}_{\mathrm{x}} f-\mathrm{J}_{\mathrm{x}_{8}} f<\epsilon .
$$

Now $f$ is limited in $\mathfrak{A}_{8}$ and we can take $\lambda$ so great that $f<\lambda$ in $\mathfrak{A}_{\delta}$. Then

$$
\mathrm{J}_{\mathrm{x}_{\delta}} f-\nabla_{\mathrm{x}_{\delta}} f_{\lambda}=0, \quad 0 \leqq \mathrm{~J}_{x^{\prime} \delta} f-\nabla_{x^{\prime} \delta} f_{\lambda}<\epsilon .
$$

Adding and using theorem 8,

Hence $\nabla$ exists and $J=\nabla$.

$$
0 \leqq \mathrm{~J}_{\mathrm{n}} f-\nabla_{\mathrm{n}} f_{\lambda}<\epsilon .
$$

In case $f<0$, similar reasoning applies. If $f$ is both positive and negative in $\mathfrak{A}$, set $f=\phi-\psi$ as above. Then

$$
J_{x} \phi=\nabla_{x} \phi, \quad J_{*} \psi=\nabla_{x} \psi .
$$

Subtracting and using theorem 9 , we obtain $\mathrm{J}_{x} f=\nabla_{*} f$.

Theorem 17. If $\nabla$ exists, then $\mathrm{J}$ exists and $\nabla=\mathrm{J}$.

Assume as in the preceding theorem, $f \geqq 0$. The other cases present no new difficulty. By definition we can find $\lambda_{0}$ so great that for each $\lambda>\lambda_{0}, \lambda^{\prime}>\lambda_{0}$,

$$
\nabla_{x_{1}} f=\nabla_{x_{g}^{\prime}} f_{\lambda}+\nabla_{x_{\delta}} f_{\lambda^{\prime}}+\epsilon^{\prime} \quad\left(0<\varepsilon^{\prime} \leqq \frac{1}{2} \varepsilon\right) .
$$

This relation holds for all divisions $\Delta$ since $\mathfrak{A}_{\delta}$ and $\mathfrak{A}_{\delta^{\prime}}$ are unmixed aggregates. Fix $\lambda$, and take a fixed division $\Delta$ of norm $\delta$ so small that

$$
\nabla_{x_{\delta}^{\prime}}<\frac{\epsilon}{2} .
$$

Choose further, $\lambda^{\prime}$ greater than the maximum of $f^{\prime}$ in $\mathfrak{A}_{\delta}$. Then

$$
\nabla_{\mathrm{x}_{8}} f_{\lambda^{\prime}}=\mathrm{J}_{\mathrm{x}_{8}} f \text {. }
$$

problem to great advantage. In general the proof consists in a simple paseage to the limit. Space will permit the statement of a few properties only. $1^{\circ}$. Let $f(x) \geqq M$ in a measurable field $\mathfrak{A}$. Then $\nabla_{\mathfrak{X}} f>M \mathfrak{A}$. $2^{\circ}$. Given $0<M \leqq f$ for all points of $\mathfrak{A}$. If $f$ is integrable; then $1 / f$ is integrable. $3^{\circ}$. Let $f$ be integrable in $2 t$ und let $g$ le integrable and have one sign. If $g$ is numerically less than some fuxed number $M$, then $\left|\nabla_{\mathfrak{x}} f g\right| \leqq M\left|\nabla_{\mathfrak{n}} f\right| . \quad 4^{\circ}$. Let $f$ and $g$ be integrable in $\mathfrak{A}$ and have no point of infinite discontinuity in common. Then $f g$ is integrable. $5^{\circ}$. If $f \geqq g$, then $\nabla f \geqq \nabla g . \quad 6^{\circ}$. Let $f$ be integrable in $\mathfrak{A}$ and not negative, zohile $g$ is limited and integrable. Let $\theta$ denote a mean value of $g$. Then $\nabla \mathfrak{x} f g=\theta \nabla_{:} f .7^{\circ}$. Let $f$ be integrable and not negative. Let $g$ be a continuous function of $x_{1}, \cdots, x_{m}$. Let $x_{1}, \cdots, x_{m}$ be continuous functions of a paruneter Then $g=\Phi(t)$ and $\nabla f \cdot g=\Phi(\bar{t}) \nabla f$ where $\bar{t}$ is a mean value of $t$.

* Loo. cit., p. 158. 
From (1), (2), and (3),

and the theorem is established.

$$
\nabla_{*} f-\mathrm{J}_{*_{\delta}} f<\epsilon
$$

\section{§ 3. Tests for convergence of singular integrals.}

In many cases, the problem in regard to an integral is solved when it is determined whether, in the neighborhood of certain points, the integral converges. Any method which will obviate the laborious process of a direct integration will have considerable application in practical work. Various tests may be devised for the generalized integral under discussion, some of which are given here.

Method I. If

$$
I_{\lambda_{1} \lambda_{2}}=\int_{* 1} f_{\lambda_{1} \lambda_{2}}
$$

may be developed by Taylor's expansion (in finite form or otherwise)

$$
\begin{aligned}
I_{\lambda_{1} \lambda_{g}}=\lambda_{1}^{m_{1}}\left[\phi _ { 1 } \left(x_{1}, \cdots,\right.\right. & \left.\left.x_{m}\right)\right]+\lambda_{1}^{m_{1}-1}\left[\phi_{2}\left(x_{1}, \cdots, x_{m}\right)\right]+\cdots \\
& +\lambda_{2}^{m_{2}}\left[\psi_{1}\left(x_{1}, \cdots, x_{m}\right)\right]+\lambda_{2}^{m_{2}-1}\left[\psi_{2}\left(x_{1}, \cdots, x_{m}\right)\right]+\cdots,
\end{aligned}
$$

where $\phi_{1}$ and $\psi_{1}$ are limited functions, independert of $\lambda_{1}$ and $\lambda_{2}$, the follouing criterion may at once be written down.

$$
\begin{aligned}
& \text { If } n_{1}>0 \text {, or } n_{2}>0, I_{\lambda_{1} \lambda_{2}} \text { diverges ; } \\
& \text { if } n_{1} \leqq 0 \text {, and } n_{2} \leqq 0, I_{\lambda_{1} \lambda_{2}} \text { converges.* }
\end{aligned}
$$

METHOD II. Let f be regular, except in the neighborhood of an isoluted point $\alpha=\left(\alpha_{1}, \cdots, \alpha_{m}\right)$. Around the point describe un m-dimensional sphere of radius $r$. On the surface of the sphere suppose that the maximum of the absolute value of the function is $M$. Suppose further that $\mu$ is an exponent which, for all values of $r$ as small as we choose, gives the relation $M^{\mu}<G$, where $G$ is a constant. If now $\mu<m$, the function is integrable. If $M r^{\mu}>G$ and $\mu \geqq m$, the finction is not integrable.

Effect a change of variables + by means of the generalized polar transforma-

* In calculating the volume of the solid generated by turning the Witch about its asymptote as axis, $\$ 2$, the expression obtained for $I_{\lambda_{1} \lambda_{2}}$ may be developed either in finite or infinite form,

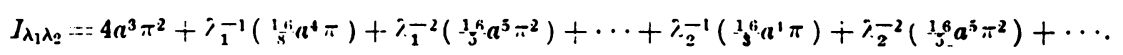

Since $n_{1}=0, n_{2}=0, J_{\lambda_{1} \lambda_{2}}$ converges.

†The equations are

$$
\begin{gathered}
x_{1}=\rho \cos \theta_{1}, x_{2}=\rho \sin H_{1} \cos \theta_{2}, x_{3}=\rho \sin \theta_{1} \sin \theta_{2} \cos \theta_{3}, \cdots \\
x_{m-1}=\rho \sin \theta_{1} \sin H_{2} \cdots \sin \theta_{m-2} \cos \theta_{m-1}, x_{m}=\rho \sin \theta_{1} \sin \theta_{2} \cdots \sin \theta_{m-2} \sin \theta_{m-1} ;
\end{gathered}
$$

where $\theta_{m-1}$ raries from 0 to $2 \pi, H_{1}, \theta_{2}, \cdots, A_{m-2}$ from 0 to $\pi$, and $\rho$ from 0 to $\infty$. 
tion. Denote by $r$ and $r_{1}$ radii of two concentric spheres about $\alpha$, and by $\mathfrak{B}$ the aggregate contained in the hollow shell. By a simple computation

$$
\begin{aligned}
& \int_{y}|f| \leqq \frac{8 G\left[r^{n-\mu}-r_{1}^{n-\mu}\right]}{2 \cdot 4 \cdot 6 \cdots n} \cdot \frac{1}{n-\mu}\left(\frac{\pi}{2}\right)^{n / 2} \text { if } n \text { is even, } \\
& \leqq \frac{8 G\left[r^{n-\mu}-r_{1}^{n-\kappa}\right]}{3 \cdot 5 \cdot 7 \cdots n-2} \cdot \frac{1}{n-\mu}\left(\frac{\pi}{2}\right)^{(n-1) / 2} \text { if } n \text { is odd. }
\end{aligned}
$$

Suppose now $\mu<m$. Then for $r_{1}<r$,

$$
\lim _{r=0} \int_{\mathfrak{z}}|f|=0
$$

and this is a sufficient condition for the convergence of the integral.

To establish the second half of this test it is sufficient to notice that if $n \geqq \mu$ and $G_{1}$ is a constant

$$
\int_{y}|f|>G_{1}
$$

By theorem 13 the integral diverges.

As a generalization of a test used in integrals of functions of one variable, we get another important test; from another point of view it may be considered as a special case of method II.

METHOD III. Let $\alpha=\left(\alpha_{1}, \cdots, \alpha_{m}\right)$ be an isolated point of infinite discontinuity of $f\left(x_{1}, \cdots, x_{m}\right)$. Let $M>0$ be a constunt and $\mu_{1}, \cdots, \mu_{m}$ be a series of exponents each less than unity. If

$$
\left(\left|x_{1}-\alpha_{1}\right|\right)^{\mu_{1}}\left(\left|x_{2}-\alpha_{2}\right|\right)^{\mu_{2}} \cdots\left(\left|x_{m}-\alpha_{m}\right|\right)^{\mu_{m}}|f(x)|<M
$$

for $x$ in $\nabla_{\delta}(\alpha) *$ (the vicinity of $\alpha$ of norm $\delta$ ), then $f$ is integrable.

YaLe Univeresity, NeW HAVEN, December, 1905.

\footnotetext{
* For explanation of notation see Lectures, $\$ 250,1$.
} 